\title{
SOLUTIONS OF INITIAL VALUE PROBLEMS FOR A PAIR OF LINEAR FIRST ORDER ORDINARY DIFFERENTIAL SYSTEMS WITH INTERFACE-SPATIAL CONDITIONS
}

\author{
M. VENKATESULU and PALLAV KUMAR BARUAH \\ Sri Sathya Sai Institute of Higher Learning \\ Department of Mathematics and Computer Science \\ Prasanthinilayam 515134 (A.P.) INDIA
}

(Received April, 1995; Revised August, 1995)

\begin{abstract}
Solutions of initial value problems associated with a pair of ordinary differential systems $\left(L_{1}, L_{2}\right)$ defined on two adjacent intervals $I_{1}$ and $I_{2}$ and satisfying certain interface-spatial conditions at the common end (interface) point are studied.

Key words: Interface-Spatially Mixed Conditions, Ordinary Differential Systems, Equations, Initial Value Problems, Linearly Independent Solutoins, Fundamental Systems.
\end{abstract}

AMS (MOS) subject classifications: $34 \mathrm{AXX}, 34 \mathrm{~A} 10,34 \mathrm{~A} 15$.

\section{Introduction}

In the studies of acoustic waveguides in ocean [1], optical fiber transmission [4], soliton theory [3], etc., we encounter a new class of problems of the type

$$
L_{1} f_{1}=\sum_{k=0}^{n} P_{k} \frac{d f_{1}^{k}}{d t^{k}}=\theta f_{1} \text { defined on an interval } I_{1}
$$

and

$$
L_{2} f_{2}=\sum_{k=0}^{m} Q_{k} \frac{d f_{2}^{k}}{d t^{k}}=\theta_{2} f_{2} \text { defined on an adjacent interval } I_{2}
$$

where $\theta_{1}, \theta_{2}$ are constants, intervals $I_{1}$ and $I_{2}$ have common end (interface) point $t=c$, and the functions $f_{1}, f_{2}$ are required to satisfy certain interface conditions at $t=c$. In most of the cases, the complete set of physical conditions on the system gives rise to self adjoint eigenvalue problems associated with the pair $\left(L_{1}, L_{2}\right)$. In some cases, however, the physical conditions at the interface may be inadequate to describe the problem in a mathematically sound manner. In such a situation, when the problem is formulated mathematically, it becomes ill-posed, and therefore cannot be solved effectively (uniquely) using existing methods. With the introduction of interface-spatial conditions (entirely a new concept), we shall be able to convert these ill-posed problems into wellposed problems and this justifies their mathematical study.

In a series of papers, we wish to develop a unified approach to these interface-spatial problems for both the regular and the singular cases. In the present paper, for the first time, we 
shall study the initial value problems (IVPs) for a pair of linear first order ordinary differential systems satisfying certain interface-spatial conditions.

Before proving the main theorems, we introduce a few notations and make some assumptions. For any compact interval $J$ of $\mathbb{R}$ and for any non-negative integer $k$, let $C^{k}(J)$ denote the space of $k$-times continuously differentiable complex-valued functions defined on $J$. If $I$ is a non-compact interval of $\mathbb{R}, C^{k}(I)$ denotes the collection of all complex-valued functions $f$ defined on $I$ whose restriction $\left.f\right|_{J}$ to any compact subinterval $J$ of $I$ belongs to $C^{k}(J)$. Let $A C^{k}(I)$ denote the space of all complex-valued functions $f$ which have $(k-1)$ derivatives on $I$, and, the $(k-t)^{t h}$ derivative is absolutely continuous over each compact subinterval of $I$. Let $I_{1}=(a, c], I_{2}=[c, b)$, $-\infty \leq a<c<b \leq+\infty$, and let $f^{(j)}$ denote the $j^{t h}$ derivative of $f$. For a matrix $A$, let $R(A)$ and $\rho(A)$ denote the range and rank of $A$. Let $\mathbb{C}^{n}$ denote the complex $n$-dimensional space.

Let $A_{f}(t)\left(A_{2}(t)\right)$ be matrix valued functions of order $n \times n(m \times m)$, whose entries belong to $\mathbb{C}^{0}\left(I_{1}\right)\left(\mathbb{C}^{0}\left(I_{2}\right)\right)$. Let $b_{1}(t)\left(b_{2}(t)\right)$ be a vector-valued function of order $n \times 1(m \times 1)$, whose entries are integrable over every compact subinterval of $I_{1}\left(I_{2}\right)$.

Let the functions $P_{k} \in \mathbb{C}^{k}\left(I_{1}\right) \quad(k=0,1, \ldots, n) Q_{k} \in \mathbb{C}^{k}\left(I_{2}\right) \quad(k=0,1, \ldots, m) P_{n}(t) \neq \emptyset$ on $I_{1}$ and $Q_{m}(t) \neq \emptyset$ on $I_{2}$. Let $g_{1}\left(g_{2}\right)$ be a measurable complex-valued function defined on $I_{1}\left(I_{2}\right)$ which is integrable over every compact subinterval of $I_{1}\left(I_{2}\right)$.

Without loss of generality, we assume $n \geq m$. Let $A$ and $B$ be $m \times n$ and $m \times m$ matrices with complex entries respectively, and $R(A)=R(B)$. Consequently, $\rho(A)=\rho(B)=: d(\leq m)$. Let $N$ be a subspace of $R(A)$, and the dimension of $N$ equals $d^{\prime}$. Let $t_{i} \in I_{i} \quad(i=1,2)$, $C=$ column $\left(c_{0}, c_{1}, \ldots, c_{n-1}\right) \in \mathbb{C}^{n}$, and $D=\operatorname{column}\left(d_{0}, d_{1}, \ldots, d_{m-1}\right) \in \mathbb{C}^{m}$. Let $Y_{1}=$ column $\left(y_{11}, y_{12}, \ldots, y_{1 n}\right)$ and $Y_{2}=\operatorname{column}\left(y_{21}, y_{22}, \ldots, y_{2 m}\right)$.

Consider the following interface-spatially mixed pair of linear first order ordinary differential systems:

$$
\begin{gathered}
Y_{1}^{\prime}=A_{1}(t) Y_{1}+b_{1}(t), t \in I_{1}, \\
Y_{2}^{\prime}=A_{2}(t) Y_{2}+b_{2}(t), \quad t \in I_{2}, \\
A Y_{1}(c)-B Y_{2}(c) \in N .
\end{gathered}
$$

Also, consider the initial conditions

and

$$
Y_{1}(c)=C
$$

$$
Y_{2}(c)=D
$$

We call problems (1)-(3) and (4)((5)) the interface-spatially mixed initial value problems $(I F S I V P)(I)((I I))$.

Consider the following interface-spatially mixed pair of linear ordinary differential equations (of orders $n$ and $m$ ):

where

$$
\begin{gathered}
L_{1} f_{1} \equiv \sum_{k=0}^{n} P_{k} \frac{d^{2} f_{1}}{d t^{2}}=g_{1}, \quad t \in I_{1} \\
L_{2} f_{2} \equiv \sum_{k=0}^{m} Q_{k} \frac{d^{2} f_{2}}{d t^{2}}=g_{2}, \quad t \in I_{2} \\
A \tilde{f}_{1}(c)-B \tilde{f}_{2}(c) \in N
\end{gathered}
$$

$$
\tilde{f}_{1}=\operatorname{column}\left(f_{1}, f_{1}^{(1)}, \ldots, f_{1}^{(n-1)}\right.
$$


and

$$
\tilde{f}_{2}=\operatorname{column}\left(f_{2}, f_{2}^{(1)}, \ldots, f_{2}^{(m-1)}\right) .
$$

Also consider the initial conditions

$$
\begin{aligned}
& f_{1}^{(j)}\left(t_{1}\right)=c_{j} \quad(j=0,1, \ldots, n-1), \\
& f_{2}^{(j)}\left(t_{2}\right)=d_{j} \quad(j=0,1, \ldots, m-1) .
\end{aligned}
$$

We call problems (6)-(8) and (9) ((10)) the interface-spatially mixed initial value problems (IFSIVP) $\left(I^{\prime}\right)\left(\left(I I^{\prime}\right)\right)$.

Definition 1: We call a pair of vector-valued functions $\left(Y_{1}, Y_{2}\right)$, defined on $I_{1} \times I_{2}$, an interface-spatially mixed (IFS) solution of (1)-(2) if

(i) $\quad Y_{1 j} \in A C^{1}\left(I_{1}\right)(j=1, \ldots, n)$,

(ii) $\quad Y_{1}$ satisfies equation (1) for almost all $t \in I_{1}$,

(iii) $Y_{2 j} \in A C^{1}\left(I_{2}\right) \quad(j=1, \ldots, m)$, and

(iv) $Y_{2}$ satisfies equation (2) for almost all $t \in I_{2}$,

(v) the pair $\left(Y_{1}, Y_{2}\right)$ satisfies relation (3).

Definition 2: We call a pair of complex-valued functions $\left(f_{1}, f_{2}\right)$, defined on $I_{1} \times I_{2}$, an interface-spatially mixed (IFS) solution of (6)-(7) if

(i) $f_{1} \in A C^{n}\left(I_{1}\right)$ and satisfies equation (6) for almost all $t \in I_{1}$,

(ii) $f_{2} \in A C^{m}\left(I_{2}\right)$ and satisfies equation (7) for almost all $t \in I_{2}$ and

(iii) the pair $\left(f_{1}, f_{2}\right)$ satisfies relation (8).

Definition 3: We call a pair of vector-valued functions $\left(Y_{1}, Y_{2}\right)$, defined on $I_{1} \times I_{2}$, an interface-spatially mixed solution of IFSIVP $(I)((I I))$ if

(i) $\quad\left(Y_{1}, Y_{2}\right)$ is an IFS solution of (1)-(2) and

(ii) $\quad Y_{1}\left(Y_{2}\right)$ satisfies condition (4) ((5)).

Definition 4: We call a pair of complex-valued functions $\left(f_{1}, f_{2}\right)$, defined on $I_{1} \times I_{2}$, an interface-spatially mixed solution of $\operatorname{IFSIVP}\left(I^{\prime}\right)\left(\left(I I^{\prime}\right)\right)$ if

(i) $\quad\left(f_{1}, f_{2}\right)$ is a IFS solution of $(6)-(7)$ and

(ii) $\quad\left(f_{1}, f_{2}\right)$ satisfies condition $(9)((10))$.

Definition 5: We say that a collection of non-trivial pairs $\left(Y_{11}, Y_{12}\right), \ldots,\left(Y_{p 1}, Y_{p 2}\right)$ are linearly independent if for any set of scalars $\alpha_{1}, \ldots, \alpha_{p}$,

$$
\sum_{i=0}^{p} \alpha_{i}\left(Y_{i 1}, Y_{i 2}\right)=(0,0)
$$

implies that $\alpha_{1}=\alpha_{2}=\ldots=\alpha_{p}=0$.

Similarly, we define the linear independency of a collection of pairs $\left(f_{11}, f_{12}\right), \ldots,\left(f_{p 1}, f_{p 2}\right)$.

Definition 6: By an IFS fundamental system for the IFSIVP $(I)((I I))$, we mean a set of linearly independent IFS solutions of IFSIVP $(I)((I I))$ which span the IFS solution space of $\operatorname{IFSIVP}(I)((I I))$.

Similarly, we define a fundamental system for the $\operatorname{IFSIVP}\left(I^{\prime}\right)\left(\left(I I^{\prime}\right)\right)$. 


\section{Main Theorems}

Theorem 1: $(a)$ If either $b_{1}(t) \neq 0, b_{2}(t) \neq 0$, or $C$ is a nonzero vector, then the IFSVP $(I)$ has an IFS fundamental system consisting of " $m-d+d^{\prime}+1$ " linearly independent IFS solutions of $\operatorname{IFSIVP}(I)$. If $b_{1}(t) \equiv 0, b_{2}(t) \equiv 0$, and $C$ is a zero vector, then the IFSIVP $(I)$ has an IFS fundamental system consisting of " $m-d+d$ " linearly independent IFS solutions of IFSIVP(I).

(b) If either $b_{1}(t) \neq 0, b_{2}(t) \neq 0$, or $D$ is a nonzero vector, then the IFSIVP(II) has a fundamental system consisting of " $n-d+d$ ' +1 " linearly independent IFS solutions of $\operatorname{IFSIVP}(I I)$. If $b_{1}(t) \equiv 0, b_{2}(t)=0$, and $D$ is a zero vector, then the IFSIVP $(I I)$ has an IFS fundamental system consisting of " $n-d+d$ " linearly independent IFS solutions of IFSIVP(II).

Proof: Since the components of $b_{1}(t)$ are measurable complex-valued functions integrable on $I_{1}$ by Theorem 2.1 [2], there exists a unique vector-valued function $\phi(t)=\operatorname{column}\left(\phi_{1}(t)\right.$, $\left.\phi_{2}(t), \ldots, \phi_{n}(t)\right)$ defined on $I_{1}$ with $\phi_{j} \in A C^{1}\left(I_{1}\right)$ such that

$$
\begin{gathered}
\phi^{\prime}(t)=A_{1}(t) \phi(t)+b_{1}(t), t \in I_{1}, \\
\phi\left(t_{1}\right)=C .
\end{gathered}
$$

Let $\phi(c)=\eta$. Since $R(A)=R(B)$, there exists a vector $\beta^{0} \in \mathbb{C}^{m}$ such that $A \eta=B \beta^{0}$. If $A \eta \neq 0, \beta^{0}$ is a nonzero vector, and if $A \eta=0$, then we take $\beta^{0}$ to be zero vector. Since $\rho(B)=d$, there exist $(m-d)$ linearly independent vectors $\beta^{1}, \beta^{2}, \ldots, \beta^{m-d} \in \mathbb{C}^{m}$ which are solutions of $B \beta=0$. Clearly, $\beta^{0}, \beta^{0}+\beta^{1}, \ldots, \beta^{0}+\beta^{m-d}$ are $(m-d+1)$ or $(m-d)$ linearly independent solutions of $A \eta=B \beta$, affected by $A \eta \neq 0$ or $A \eta=0$.

Also, since the components of $b_{2}(t)$ are measurable complex-valued functions integrable on $I_{2}$, there exists a unique vector-valued function $\psi_{0}(t)=$ column $\left(\psi_{01}(t), \ldots, \psi_{0 n}(t)\right)$ defined on $I_{2}$ with $\psi_{0 j}(t) \in A C^{1}\left(I_{2}\right)$ such that

$$
\begin{gathered}
\psi^{\prime}(t)=A_{2}(t) \psi_{0}(t)+b_{2}(t), \quad t \in I_{2} \\
\psi_{0}(c)=\beta^{0} .
\end{gathered}
$$

Let $\psi_{i}(t)=\operatorname{column}\left(\psi_{i 1}(t), \ldots, \psi_{i n}(t)\right)$, defined on $I_{2}$ with $\psi_{i j} \in A C^{1}\left(I_{2}\right)$, be the unique vector-valued function such that

$$
\begin{aligned}
& \psi_{i}(t)=A_{2}(t) \psi_{i}(t), \quad t \in I_{2}, \\
& \psi_{i}(c)=\beta^{i}, \quad i=1, \ldots, m-d .
\end{aligned}
$$

Clearly, $\psi_{1}, \ldots, \psi_{m-d}$ are linearly independent and if $\beta^{0} \neq 0$, then $\psi_{0}, \ldots, \psi_{m-d}$ are also linearly independent.

Choose a basis $\alpha^{1}, \ldots, \alpha^{d^{\prime}}$ for $N$, and let $\beta=\beta^{m-d+i}$ be a solution of

$$
-B \beta^{m-d+i}=\alpha^{i} \quad\left(i=1, \ldots, d^{\prime}\right)
$$

Since $\alpha^{i}$ are linearly independent $\beta^{m-d+d^{\prime}} s$ are also linearly independent. In fact, $\beta^{1}, \ldots, \beta^{m-d+d^{\prime}}$ are linearly independent.

Again, let $\psi_{i}(t)$, defined on $I_{2}$, be a unique vector-valued function such that

$$
\begin{gathered}
\psi_{i}(t)=A_{2}(t) \psi_{i}(t), \quad t \in I_{2}, \\
\psi_{i}(c)=\beta^{i}\left(i=m-d+1, \ldots, m-d+d^{\prime}\right) .
\end{gathered}
$$

Clearly, $\psi_{1}, \ldots, \psi_{m-d+d^{\prime}}$ are linearly independent.

Now, define

$$
\left(Y_{01}, Y_{02}\right)=\left(\phi, \psi_{0}\right)
$$




$$
\left(Y_{i 1}, Y_{i 2}\right)=\left(\phi, \psi_{0}+\psi_{i}\right) \quad\left(i=1, \ldots, m-d+d^{\prime}\right) .
$$

Clearly, each pair $\left(Y_{i 1}, Y_{i 2}\right) \quad\left(i=0,1, \ldots, m-d+d^{\prime}\right)$ is an IFS solution of (1)-(2). Moreover, if $b_{1}(t) \neq 0, b_{2}(t) \neq 0$, or $C \neq 0$, then the pair $\left(\phi, \psi_{0}\right)$ is nontrivial.

Claim: For $b_{1}(t) \neq 0, b_{2} \neq 0$ or $C \neq 0,\left\{\left(Y_{i 1}, Y_{i 2}\right), i=0, \ldots, m-d+d^{\prime}\right\}$ is an IFS fundamental system for the IFSIVP $(I)$.

Let $\sum_{i=0}^{m-d+d^{\prime}} a_{i}\left(Y_{i 1}, Y_{i 2}\right)=(0,0)$, where $a_{i} s$ are scalars. Then

Consequently, we get

$$
\sum_{i=0}^{m-d+d^{\prime}} a_{i} Y_{i 1}=0 \text { and } \sum_{i=0}^{m-d+d^{\prime}} a_{i} Y_{i 2}=0 .
$$

$$
\begin{gathered}
\sum_{i=1}^{m-d+d^{\prime}} a_{i}\left[A \phi(c)-B\left(\psi_{0}(c)+\psi_{i}(c)\right)\right]+a_{0}\left(A \phi(c)-B \psi_{0}(c)\right)=0 \\
\sum_{i=m-d+i}^{m-d^{\prime}} a_{i}\left(-B \psi_{i}(c)\right)=0
\end{gathered}
$$

i.e.,

$$
\sum_{i=m-d+1}^{m-d+d^{\prime}} a_{i} \alpha^{i}=0, \text { which implies that } a_{i}=0 \quad\left(i=m-d+1, \ldots, m-d+d^{\prime}\right) .
$$

Hence, relation (11) becomes

$$
\sum_{i=0}^{m-d} a_{i} \phi=0 \text { and } \sum_{i=1}^{m-d} a_{i}\left(\psi_{0}+\psi_{i}\right)+A_{0} \psi_{0}=0 .
$$

Again, from relation (12), we get

$$
\left(\sum_{i=0}^{m-d} a_{i}\right) \psi_{0}(c)+\sum_{i=1}^{m-d} a_{i} \psi_{i}(c)=0
$$

i.e.,

$$
\left(\sum_{i=0}^{m-d} a_{i}\right) \beta^{0}+\sum_{i=1}^{m-d} a_{i} \beta^{i}=0
$$

If $\beta^{0} \neq \emptyset$, then $\beta^{0}, \beta^{1}, \ldots, \beta^{m-d}$, are linearly independent and hence $a_{i}=0(i=0,1, \ldots, m-d)$. If $\beta^{0}=0$, then relation (13) gives $a_{i}=0(i=1, \ldots, m-d)$ and from relation (12) we get $a_{0}\left(\phi, \psi_{0}\right)=(0,0)$, which implies that $a_{0}=0$. Thus, $\left(Y_{i 1}, Y_{i 2}\right) \quad\left(i=0,1, \ldots, m-d+d^{\prime}\right)$ are linearly independent.

Now, let $\left(Y_{1}, Y_{2}\right)$ be any solution of the $\operatorname{IFSIVP}(I)$. We note that $Y_{1}=\phi$.

Case (i): Suppose that $A Y_{1}(c)-B Y_{2}(c)=0$. Furthermore, since $A \phi(c)-B \psi_{0}(c)=0$, we get $B\left(Y_{2}(c)-\psi_{0}(c)\right)=0$, which implies that $Y_{2}(c)-\psi_{0}(c)$ belongs to the null space of $\mathrm{B}$. Therefore, there exist constants $a_{i}(i=1, \ldots, m-d)$ such that

i.e.,

$$
Y_{2}(c)-\psi_{0}(c)=\sum_{i=1}^{m-d} a_{i} \beta^{i}
$$

$$
\begin{aligned}
Y_{2}(c)= & \beta^{0}+\sum_{i=1}^{m-d} a_{i} \beta^{i}=\left(1-\sum_{i=1}^{m-d} a_{i}\right) \beta^{0}+\sum_{i=1}^{m-d} a_{i}\left(\beta^{0}+\beta^{i}\right) \\
= & \left(1-\sum_{i=1}^{m-d} a_{i}\right) \psi_{0}(c)+\sum_{i=1}^{m-d} a_{i}\left(\psi_{0}(c)+\psi_{i}(c)\right) \\
= & \left(1-\sum_{i=1}^{m-d} a_{i}\right) Y_{02}(c)+\sum_{i=1}^{m-d} a_{i} Y_{i 2}(c) .
\end{aligned}
$$


Thus, by the uniqueness of the solution of IVPs for a system of ordinary differential equations, we have

$$
\left(Y_{1}, Y_{2}\right)=\left(1-\sum_{i=1}^{m-d} a_{i}\right)\left(Y_{01}, Y_{02}\right)+\sum_{i=1}^{m-d} a_{i}\left(Y_{i 1}, Y_{i 2}\right) \text {. }
$$

Case (ii): Suppose that $A Y_{1}(c)-B Y_{2}(c)=\xi=\sum_{i=1}^{d^{\prime}} a_{i+m-d} \alpha_{i}$, where $a_{i} s$ are scalars.

Define a pair $\left(K_{1}, K_{2}\right)$ by

$$
\left.\left(K_{1}, K_{2}\right)=\underset{i=m-d+1}{\left(1-\sum_{i}^{m-d+d^{\prime}}\right.} a_{i}\right)\left(Y_{01}, Y_{02}\right)+\sum_{i=m-d+1}^{m-d+d^{\prime}} a_{i}\left(Y_{i 1}, Y_{i 2}\right) .
$$

Then $\left(K_{1}, K_{2}\right)$ is an IFS solution of $\operatorname{IFSIVP}(I)$. Consequently, we get

$$
B\left(Y_{2}(c)-K_{2}(c)\right)=0 .
$$

Therefore, there exist scalars $a_{i}(i=1, \ldots, m-d)$ such that

i.e.,

$$
\begin{gathered}
Y_{2}(c)-K_{2}(c)=\sum_{i=1}^{m-d} a_{i} \beta^{i} \\
Y_{2}(c)=K_{2}(c)+\sum_{i=1}^{m-d} a_{i} \beta^{i} \\
=K_{2}(c)-\left(\sum_{i=1}^{m-d} a_{i}\right) \beta^{0}+\sum_{i=1}^{m-d} a_{i}\left(\beta^{0}+\beta^{i}\right) \\
=K_{2}(c)-\left(\sum_{i=1}^{m-d} a_{i}\right) \psi_{0}(c)+\sum_{i=1}^{m-d} a_{i}\left(\psi_{0}(c)+\psi_{i}(c)\right)
\end{gathered}
$$

Thus,

$$
\begin{gathered}
\left(Y_{1}, Y_{2}\right)=\left(K_{1}, K_{2}\right)-\sum_{i=1}^{m-d} a_{i}\left(Y_{01}, Y_{02}\right)+\sum_{i=1}^{m-d} a_{i}\left(Y_{i 1}, Y_{i 2}\right) \\
=\left(1-\sum_{i=1}^{m-d+d^{\prime}} a_{i}\right)\left(Y_{01}, Y_{02}\right)+\sum_{i=1}^{m-d+d^{\prime}} a_{i}\left(Y_{i 1}, Y_{i 2}\right) .
\end{gathered}
$$

Hence, the claim is proved. If $b_{1}(t) \equiv 0, b_{2}(t) \equiv 0$, and $C=0$, then $\left(\phi, \psi_{0}\right)$ is a trivial pair and the pairs $\left(Y_{i 1}, Y_{i 2}\right)\left(i=1, \ldots, m-d+d^{\prime}\right)$ form an IFS fundamental system for the IFSIVP $(I)$.

This completes the proof of part $(a)$. Part $(b)$ can proved similarly.

Theorem 2: There exist exactly " $n+m-d+d$ " linearly independent (IFS) solutions of

$$
\begin{array}{ll}
Y_{1}^{\prime}=A_{1}(t) Y_{1}, & t \in I_{1}, \\
Y_{2}^{\prime}=A_{2}(t) Y_{2}, & t \in I_{2},
\end{array}
$$

satisfying the interface-spatial conditions

$$
A Y_{1}(c)-B Y_{2}(c) \in N
$$

Proof: Since $\rho(A)=\rho(B)=: d$, there exists a basis $\left\{\eta^{1}, \ldots, \eta^{n}\right\}$ for $\mathbb{C}^{n}$ such that $\left\{\eta^{1}, \ldots, \eta^{n-d}\right\}$ forms a basis for the null-space of $A$, and a basis $\left\{\beta^{1}, \ldots, \beta^{m}\right\}$ for $\mathbb{C}^{m}$ such that $\left\{\beta^{d+1}, \ldots, \beta^{m}\right\}$ forms a basis for the null space of $B$.

Let $\widehat{Y}_{i 1}$ (whose components belong to $A C^{1}\left(I_{1}\right)$ ) be the unique solution of 


$$
\begin{gathered}
Y_{1}^{\prime}=A_{1}(t) Y_{1}, \quad t \in I_{1}, \\
Y_{1}(c)=\eta^{i} \quad(i=1, \ldots, n) .
\end{gathered}
$$
that

Since $R(A)=R(B)$, for each $i=n-d+1, \ldots, n$, there exist scalars $\theta_{j}^{i} \quad(j=1, \ldots, d)$ such

$$
A \eta^{i}=\sum_{j=1}^{d} \theta_{j}^{i} B \beta^{j}
$$

Let $\widehat{Y}_{i 2}$ (with components belonging to $A C\left(I_{2}\right)$ ) be the unique solution of

$$
\begin{gathered}
Y_{2}^{\prime}=A_{2}(t) Y_{2}, t \in I_{2}, \\
Y_{2}(c)=B \beta^{i-n+d}(i=n+1, \ldots, n+m-d) .
\end{gathered}
$$

Let $\left(\alpha^{1}, \ldots, \alpha^{d^{\prime}}\right\}$ be a basis for $N$ and choose $\widehat{\beta}^{i} \in \mathbb{C}^{m}$ such that

$$
-B \widehat{\beta}^{i}=\alpha^{i} \quad\left(i=1, \ldots, d^{\prime}\right) .
$$

Let $\widehat{Y}_{i 2}$ (with components belonging to $A C^{1}\left(I_{2}\right)$ ) be the unique solution of

$$
\begin{gathered}
Y_{2}^{\prime}=A_{2}(t) Y_{2}, \quad t \in I_{2}, \\
Y_{2}(c)=\widehat{\beta}^{i-n-m+d} \quad\left(i=n+m-d+1, \ldots, n+m-d+d^{\prime}\right) .
\end{gathered}
$$

Define the pairs

$$
\left(Y_{i 1}, Y_{i 2}\right)=\left\{\begin{array}{cc}
\left(\widehat{Y}_{i 1}, 0\right) & (i=1, \ldots, n-d) \\
\left(\widehat{Y}_{i 1}, \widehat{Y}_{i 2}\right) & (i=n-d+1, \ldots, n) \\
\left(0, \widehat{Y}_{i 2}\right) & \left(i=n+1, \ldots, n+m-d+d^{\prime}\right)
\end{array}\right.
$$

Clearly each pair $\left(Y_{i 1}, Y_{i 2}\right)$ is a nontrival IFS solution of (16)-(18).

Claim: $\left(Y_{i 1}, Y_{i 2}\right) \quad\left(i=1, \ldots, n+m-d+d^{\prime}\right)$ form an IFS fundamental system for the IFS solutions of (16)-(18).

First, we shall show that the pairs $\left(Y_{i 1}, Y_{i 2}\right)$ are linearly independent. To this end, let

$$
\sum_{i=1}^{n+m-d+d^{\prime}} a_{i}\left(Y_{i 1}, Y_{i 2}\right)=(0,0), \text { where } a_{i} s \text { are scalars. }
$$

Then,

$$
\sum_{i=1}^{n} a_{i} \widehat{Y}_{i 1}=0 \text { and } \quad \sum_{i=n-d+1}^{n+m+d^{\prime}} a_{i} \widehat{Y}_{i 2}=0 .
$$

Since $\widehat{Y}_{i 1}(c) \quad\left(i=1, \ldots, n+m-d+d^{\prime}\right)$ are linearly independent, from the first equation of relation (19) we get $a_{i}=0 \quad(i=1, \ldots, n)$. Consequently, the second equation reduces to

$$
\sum_{i=n+1}^{n+d+d^{\prime}} a_{i} Y_{i 2}=0
$$

Evaluating the above expression at $t=c$ and then applying the matrix $B$ to the resulting expression, we get

$$
\sum_{i=n+m-d+1}^{n+m-d+d^{\prime}} a_{i} \alpha^{i-n-m+d}=0
$$

which implies that $a_{i}=0$, for $i=n+m-d+1, \ldots, n+m-d+d^{\prime}$. Thus, relation (20) reduces to 

$\sum_{i=n+1}^{n+d} a_{i} \widehat{Y}_{i 2}=0$, and since $\widehat{Y}_{i 2}(c)(i=n+1, \ldots, n+m-d)$ are linearly independent (this fact can
be easily verified), it follows that

$$
a_{i}=0 \quad(i=n+1, \ldots, n+m-d) .
$$

This proves the linear independency of $\left(Y_{i 1}, Y_{i 2}\right)$ s.

Next, let $\left(Y_{1}, Y_{2}\right)$ be any IFS solution of (16)-(18). Choose scalars $a_{i}(i=1, \ldots, n)$ such that

$$
Y_{1}(c)=\sum_{i=1}^{n} a_{i} \eta^{i}
$$

Case (1): Suppose that $A Y_{1}(c)-B Y_{2}(c)=0$.

Define the pair $\left(K_{1}, K_{2}\right)=\sum_{i=1}^{n} a_{i}\left(Y_{i 1} Y_{i 2}\right)$.

Then $K_{1}(c)=\sum_{i=1}^{n} a_{i} Y_{i 1}(c)=Y_{1}(c)$. Hence, $Y_{1}=K_{1}$ and $B\left(Y_{2}(c)-K_{2}(c)\right)=0$, which
plies that

i.e.,

$$
Y_{2}(c)=K_{2}(c)+\sum_{i=n+1}^{n+m-d} a_{i} \beta^{i-n+d} \text { for some scalars } a_{i} s
$$

$$
Y_{2}(c)=K_{2}(c)+\sum_{i=n+1}^{n+m-d} a_{i} Y_{i 2}(c)
$$

Thus,

$$
\begin{aligned}
\left(Y_{1}, Y_{2}\right)= & \left(K_{1}, K_{2}\right)+\sum_{i=n+1}^{n+m-d} a_{i}\left(Y_{i 1}, Y_{i 2}\right) \\
& =\sum_{i=1}^{n+m-d} a_{i}\left(Y_{i 1}, Y_{i 2}\right) .
\end{aligned}
$$

Case (2): Suppose that $A\left(Y_{1}(c)-B Y_{2}(c)=\xi=\quad \sum_{i=n+m-d+1}^{n+m-d+d^{\prime}} a_{i} \alpha^{i-n-m+d}\right.$, where $a_{i} s$
scalars. are scalars.

Define $\left(H_{1}, H_{2}\right)=\sum_{i=n+m-d+1}^{n+m-d+d^{\prime}} a_{i}\left(Y_{i 1}, Y_{i 2}\right)$.

Then $A\left(H_{1}(c)-Y_{1}^{i}(c)\right)-B\left(+H_{2}(c)-Y_{2}(c)\right)=0$, and therefore, by case (1),

$$
\left(Y_{1}-H_{1}, Y_{2}-H_{2}\right)=\sum_{i=1}^{n+m-d} a_{i}\left(Y_{i 1}, Y_{i 2}\right) \text { for some scalars } a_{i} s .
$$

Thus,

$$
\begin{aligned}
\left(Y_{1}, Y_{2}\right) & =\left(H_{1}, H_{2}\right)+\sum_{i=1}^{n+m-d} a_{i}\left(Y_{i 1}, Y_{i 2}\right) \\
& n+m-d+d^{\prime} \\
& =\sum_{i=1}^{m} a_{i}\left(Y_{i 1}, Y_{i 2}\right)
\end{aligned}
$$

This completes the proof.

Remark 1: The assumption $d^{\prime}=d$ yields that there are no explicit boundary conditions at the interface point.

If $d^{\prime}=0$, then the interface-spatial condition becomes

$$
A Y_{1}(c)-B Y_{2}(c)=0
$$

which is generally called the interface condition.

Since higher order ordinary differential equations can be converted into a system of first order 
equations, Theorems 1 and 2 yield the following results for the pair $\left(L_{1}, L_{2}\right)$ :

Theorem 3: (a) If either $g_{1} \neq 0, g_{2} \neq 0$, or $c_{0}, c_{1}, \ldots, c_{n-1}$ are not all zeros, then the $\operatorname{IFSIVP}\left(I^{\prime}\right)$ has a fundamental system consisting of " $m-d+d^{\prime}+1$ " linearly independent IFS solutions of IFSIVP $\left(I^{\prime}\right)$. If $g_{1} \equiv 0, g_{2} \equiv 0$, and $c_{0}, c_{1}, \ldots, c_{n-1}$ are all zeros, then the IFSIVP $\left(I^{\prime}\right)$ has a IFS fundamental system consisting of " $m-d+d^{\prime}$ " linearly independent solutions of $\operatorname{IFSIVP}\left(I^{\prime}\right)$.

(b) If either $g_{1} \neq 0, g_{2} \neq 0$, or $d_{0}, d_{1}, \ldots, d_{n-1}$ are not all zeros, then the IFSIVP $\left(I I^{\prime}\right)$ has a IFS fundamental system consisting of " $n-d+d^{\prime}+1$ " linearly independent IFS solutions of $\operatorname{IFSIVP}\left(I I^{\prime}\right)$. If $g_{1} \equiv 0, g_{2} \equiv 0$, and $d_{0}, d_{1}, \ldots, d_{n-1}$ are all zeros, then the IFSIVP $\left(I I^{\prime}\right)$ has an IFS fundamental system consisting of " $n-d+d^{\prime}$ " linearly independent IFS solutions of $\operatorname{IFSIVP}\left(I I^{\prime}\right)$.

Theorem 4: There exist exactly " $n+m-d+d$ " linearly independent (IFS) solutions of

$$
\begin{aligned}
& L_{1} f_{1}=0, \quad t \in I_{1}, \\
& L_{2} f_{2}=0, \quad t \in I_{2},
\end{aligned}
$$

satisfying the interface-spatial conditions

$$
A \tilde{f}_{1}(c)-B \tilde{f}_{2}(c) \in N .
$$

Remark 4: For $d^{\prime}=d$, Theorems 3 and 4 reduce to Theorems 1 and 4 of [6].

For $d^{\prime}=0$, Theorems 3 and 4 reduce to Theorems 3 and 6 of [6].

For $d^{\prime}=0$ as well as for the $(m \times n)$ matrix $A$ given by

$$
A=\left(\begin{array}{ccccccc}
1 & 0 & \ldots & 0 & 0 & \ldots & 0 \\
0 & 1 & \ldots & 0 & 0 & \ldots & 0 \\
0 & 0 & \ldots & 1 & 0 & \ldots & 0
\end{array}\right)
$$

and $B$ equal to the $(m \times m)$ identity matrix, Theorems 3 and 4 reduce to Theorems 2 and 5 of [6].

\section{Physical Examples}

Example 1 - Acoustic waveguides in ocean [1]: The following problem is encountered in the study of acoustic waves in the ocean consisting of two layers: an outer layer of finite depth and an inner layer of infinite depth:

$$
\begin{gathered}
L_{1} f_{1}=\frac{d^{2} f_{1}}{d t^{2}}+k_{1}^{2} f_{1}=\lambda f_{1}, \quad 0 \leq t \leq d_{1}, \\
L_{2} f_{2}=\frac{d^{2} f_{2}}{d t^{2}}+k_{2}^{2} f_{2}=\lambda f_{2}, \quad d_{1} \leq \leq t \leq+\infty,
\end{gathered}
$$

together with the end point conditions given by

$$
f_{1}(0)=0, \operatorname{lt}_{t \rightarrow \infty} f_{2}^{(1)}(t)=0,
$$

and the interface conditions given by 


$$
f_{1}\left(d_{1}\right)=f_{2}\left(d_{1}\right), \quad 1 / \rho_{1} f_{1}^{1}\left(d_{1}\right)=1 / \rho_{2} f_{2}^{(1)}\left(d_{1}\right)
$$

Here $\rho_{1}, \rho_{2}$ are constant densities of the two layers, $k_{1}, k_{2}$ are the constants which depend upon the frequency constant and the constant sound velocities $c_{1}, c_{2}$ of the two layers, respectively, $\lambda$ is an unknown constant, $d_{1}$ denotes the depth of the outer layer, and $f_{1}, f_{2}$ stand for the depth eigenfunctions.

In this example, the interface conditions at $t=d_{1}$ of the two layers can be written in the matrix form

$$
\left(\begin{array}{cc}
1 & 0 \\
0 & 1 / \rho_{1}
\end{array}\right)\left(\begin{array}{c}
f_{1}\left(d_{1}\right) \\
f_{1}^{(1)}\left(d_{1}\right)
\end{array}\right)=\left(\begin{array}{cc}
1 & 0 \\
0 & 1 / \rho_{1}
\end{array}\right)\left(\begin{array}{c}
f_{2}\left(d_{1}\right) \\
f_{2}^{(1)}\left(d_{1}\right)
\end{array}\right)
$$

Here $A=\left(\begin{array}{cc}1 & 0 \\ 0 & 1 / \rho_{1}\end{array}\right), B=\left(\begin{array}{cc}1 & 0 \\ 0 & 1 / \rho_{2}\end{array}\right), \operatorname{rank} A=\operatorname{rank} B=2, m=n=d=2$ and $d^{\prime}=0$.

Hence, by Theorem 3 and Remark 2, there exist a unique IFS solution for any IFSIVP associated with (22)-(23) and (25). Also, By Theorem 4 and Remark 2, there exist exactly two linearly independent IFS solutions of problems (22)-(23) and (25).

Example 2 - Optical fiber transmission [4]: In the study of wave optics of step index fiber, we encounter the following problem:

$$
\begin{aligned}
& L_{1} f_{1}=\frac{d^{2} f_{1}}{d t^{2}}+1 / t \frac{d f_{1}}{d t}+\left(\eta_{1}^{2} k_{0}^{2}-\nu^{2} / t^{2}\right) f_{1}=\beta^{2} f_{1}, \quad 0<t \leq a \\
& L_{2} f_{2}=\frac{d^{2} f_{2}}{d t^{2}}+1 / t \frac{d f_{2}}{d t}+\left(\eta_{2} k_{0}^{2}-\nu^{2} / t^{2}\right) f_{2}=\beta^{2} f_{2}, \quad a \leq t<\infty
\end{aligned}
$$

together with the interface conditions at $t=a$, given by

$$
\operatorname{lt}_{t \rightarrow 0}\left|f_{1}(t)\right|<+\infty, \operatorname{lt}_{t \rightarrow \infty}\left|f_{2}(t)\right|=0 .
$$

Here $\eta_{1}$ and $\eta_{2}$ are the refractive indices of the core and cladding, respectively, $\beta$ is the wave propagation constant, $\nu$ is an integer $k_{0}=w / c, c$ is the prorogation velocity and $w$ is the wave frequency and $f_{1}$ and $f_{2}$ are the field (electromagnetic) distributions of core and cladding, respectively.

In this example, relation (28) gives continuity conditions at $t=a$. Here $A$ and $B$ are the $2 \times 2$ identity matrices, $n=m=d=2$ and $d^{\prime}=0$. Hence, by Theorem 3 and Remark 2 , there exists a unique IFS solution for IFSIVP associated with (26)-(28). Also, by Theorem 4 and Remark 2, there exist exactly two linearly independent IFS (continuous) solutions of (26)-(28).

Example 3 - One-dimensional scattering in quantum theorem [3]: In quantum theory, the one-dimensional time-independent scattering problem with the delta function scattering potential is represented by the problem:

$$
\begin{gathered}
L_{1} f_{1}=\frac{d^{2} f_{1}}{d t^{2}}+k^{2} f_{1}=0,-\infty<t \leq 0, \\
L_{2} f_{2}=\frac{d^{2} f_{2}}{d t^{2}}+\left(k^{2}-\nu_{0}\right) f_{2}=0,0 \leq t<+\infty,
\end{gathered}
$$

together with the interface conditions given by

$$
f_{1}(0)-f_{2}(0)=0
$$




$$
f_{1}^{(1)}(0)-f_{2}^{(1)}(0)=-\nu_{0} f_{1}(0)
$$

where $k^{2}=2 m E / h^{2}, \nu_{0}$ is a constant, and the functions $f_{1}$ and $f_{2}$ are associated with the flux density of the particle of the two regions, respectively. Here, $m$ denotes the mass of the particle, $E$ denotes its total energy, and $h$ denotes the Planck constant divided by $2 \pi$. In this example, the interface conditions at $t=0$ of the two regions can be written in the matrix form

Here

$$
\left(\begin{array}{cc}
1 & 0 \\
\nu_{0} & 1
\end{array}\right)\left(\begin{array}{c}
f_{1}(0) \\
f_{1}^{(1)}(0)
\end{array}\right)=\left(\begin{array}{cc}
1 & 0 \\
0 & 1
\end{array}\right)\left(\begin{array}{c}
f_{2}(0) \\
f_{2}^{(1)}(0)
\end{array}\right) .
$$

$\operatorname{rank} A=\operatorname{rank} B=2, m=n=d=2$, and $d^{\prime}=0$.

$$
A=\left(\begin{array}{cc}
1 & 0 \\
\nu_{0} & 1
\end{array}\right), B=\left(\begin{array}{ll}
1 & 0 \\
0 & 1
\end{array}\right)
$$

Hence, by Theorem 3 and Remark 2, there exists a unique IFS solution of any IFSIVP associated with (30)-(33). Also, by Theorem 4 and Remark 2, there exist exactly two linearly independent IFS solutions of (30)-(33).

Example 4: In this illustrative example, consider the following problem:

$$
\begin{aligned}
& L_{1} f_{1}=\frac{d^{2} f_{1}}{d t^{2}}-k_{1}^{2} f_{1}=0, \quad a \leq t \leq c, \\
& L_{2} f_{2}=\frac{d^{2} f_{2}}{d t^{2}}+k_{2}^{2} f_{2}=0, \quad c \leq t \leq b,
\end{aligned}
$$

together with interface condition

and the end point conditions

$$
f_{1}(c)=f_{2}(c)
$$

$$
f_{1}(a)=0=f_{2}(b)
$$

where $k_{1}$ and $k_{2}$ are constants. Problems (34)-(37) can be thought of as the transverse vibrations of a string stretched between $a$ and $b$, fixed at $a$ and $b$, with different uniform linear densities (in the portion) between $a$ and $c$ and between $c$ and $b$, and plucked at the point $t=c$.

In this example, there is only one condition at the interface (i.e., the continuity condition), and no definite relation between the derivatives is available. Therefore, we may take

$$
f_{1}^{(1)}(c)-f_{2}^{(1)}(c)=\alpha, \quad \alpha \in \mathbb{R} .
$$

We note that relation (38) is not at all a restriction on derivatives. Consequently, relation (36) and (38) can be written as

$$
\left(\begin{array}{ll}
1 & 0 \\
0 & 1
\end{array}\right)\left(\begin{array}{c}
f_{1}(c) \\
f_{1}^{(1)}(c)
\end{array}\right)-\left(\begin{array}{ll}
1 & 0 \\
0 & 1
\end{array}\right)\left(\begin{array}{c}
f_{2}(c) \\
f_{2}^{(1)}(c)
\end{array}\right) \in N
$$

where $N=$ the linear span of $\left(\begin{array}{l}0 \\ 1\end{array}\right)$.

Here, $A=B=$ the $(2 \times 2)$ identity matrix, $n=m=d=2$, and $d^{\prime}=1$. Therefore, by Theorem 3 , there exist one or two linearly independent IFS solutions of the IFSIVP associated with problems (34)-(36) depending on whether the initial data is zero or nonzero. Also, by Theorem 4, there exist three linearly independent IFS solutions of problems (34)-(36). 
Remark 3: The results of this paper are used in studying the deficiency indices and selfadjoint boundary value problems associated with $\left(L_{1}, L_{2}\right)$ satisfying interface-spatial conditions which we shall establish elsewhere.

\section{Acknowledgement}

The authors dedicate the work to the chancellor of the Institute Bhagawan Sri Sathya Sai Baba.

\section{References}

[1] Boyles, C.A., Acoustic Waveguides, Applications to Oceanic Sciences, Wiley, New York 1984.

[2] Brauer, F. and Nohel, J.A., The Qualitative Theory of Differential Equations - An Introduction, Benjamin, New York 1969.

[3] Lamb, Jr., G.L., Elements of Soliton Theory, Wiley, New York 1980.

[4] Noda, K., Optical Fiber Transmission, Studies in Telecommunication 6 (ed. by K. Noda), North-Holland, Amsterdam 1986.

[5] Venkatesulu, M. and Bhaskar, T.G., Self-adjoint boundary value problems associated with a pair of mixed linear ordinary differential equations, J. Math. Anal. Appl. 144:2 (1989), $322-341$.

[6] Venkatesulu, M. and Bhaskar, T.G., Solutions of initial value problems associated with a pair of mixed linear ordinary differential equations, J. Math. Anal. Appl. 148:1 (1990), 6378 . 


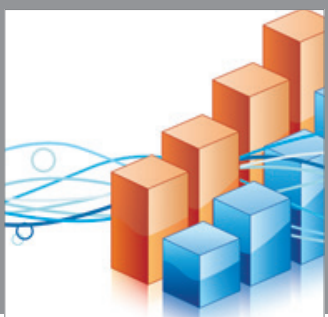

Advances in

Operations Research

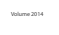

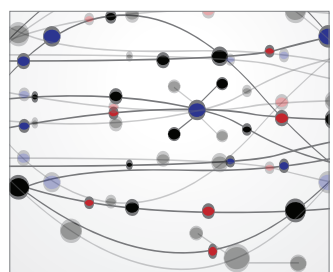

\section{The Scientific} World Journal
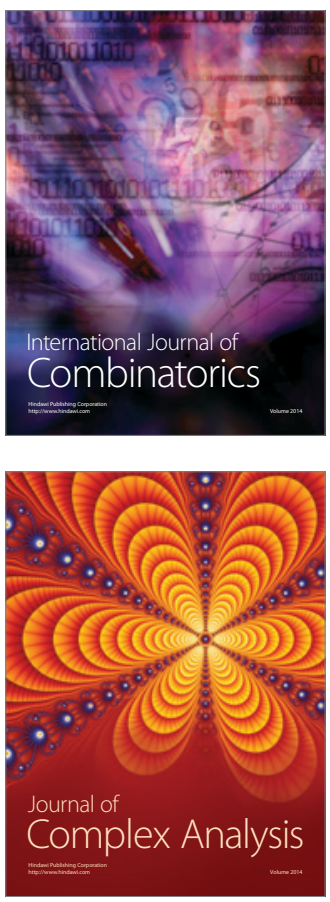

International Journal of

Mathematics and

Mathematical

Sciences
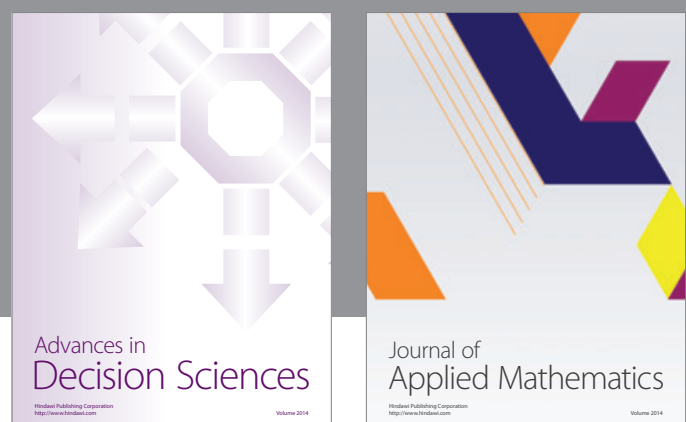

Journal of

Applied Mathematics
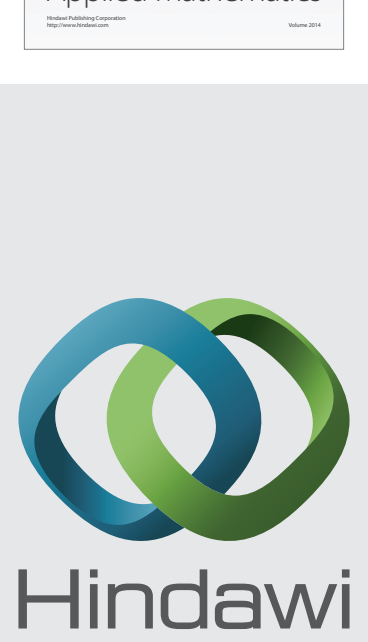

Submit your manuscripts at http://www.hindawi.com
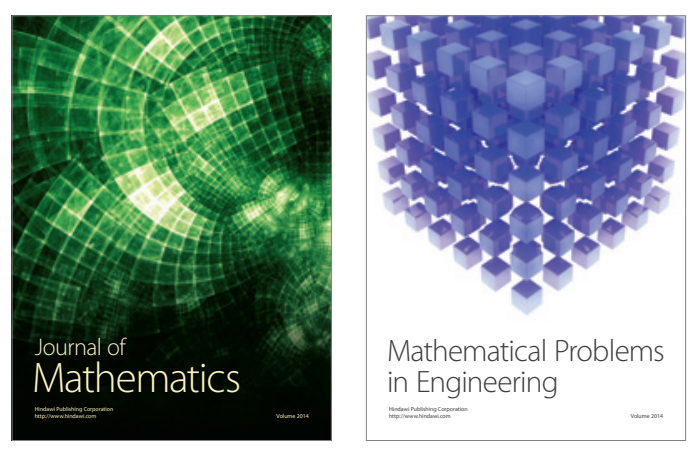

Mathematical Problems in Engineering
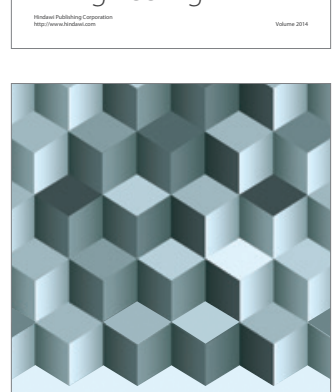

Journal of

Function Spaces
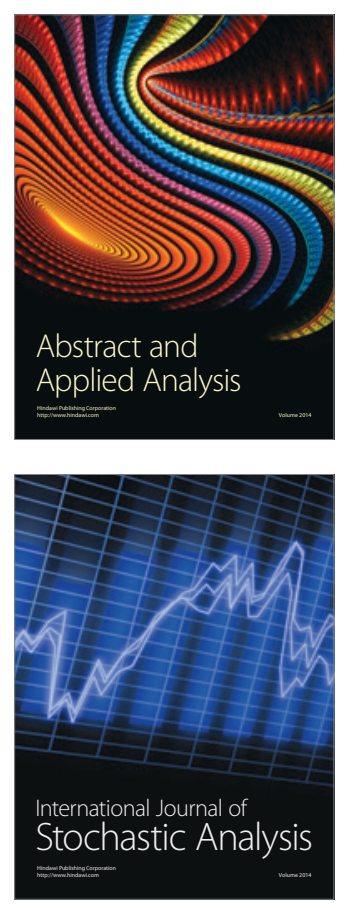

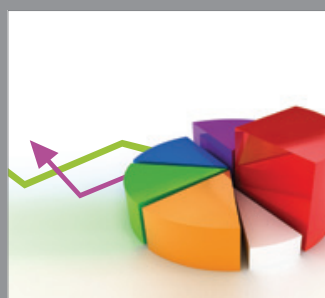

ournal of

Probability and Statistics

Promensencen
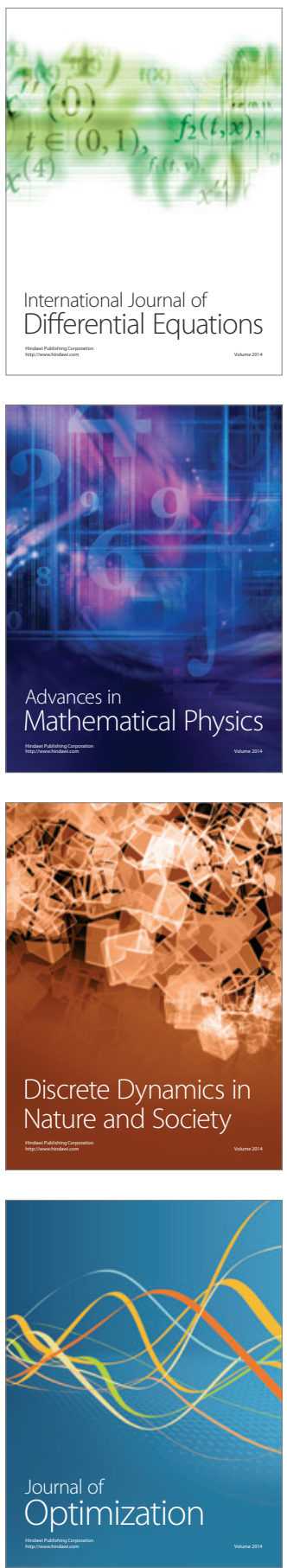